\title{
Dysphagia Management in Acute and Sub-acute Stroke
}

\author{
Alicia Vose $\cdot$ Jodi Nonnenmacher $\cdot$ Michele L. Singer • \\ Marlís González-Fernández
}

Published online: 16 September 2014

(C) Springer Science + Business Media New York 2014

\begin{abstract}
Swallowing dysfunction is common after stroke. More than $50 \%$ of the 665,000 stroke survivors will experience dysphagia acutely of which approximately 80,000 will experience persistent dysphagia at 6 months. The physiologic impairments that result in post-stroke dysphagia are varied. This review focuses primarily on well-established dysphagia treatments in the context of the physiologic impairments they treat. Traditional dysphagia therapies including volume and texture modifications, strategies such as chin tuck, head tilt, head turn, effortful swallow, supraglottic swallow, super-supraglottic swallow, Mendelsohn maneuver and exercises such as the Shaker exercise and Masako (tongue hold) maneuver are discussed. Other more recent treatment interventions are discussed in the context of the evidence available.
\end{abstract}

Keywords Stroke $\cdot$ Dysphagia $\cdot$ Swallowing $\cdot$ Deglutition . Treatment

\section{Introduction}

More than $50 \%$ of stroke survivors will experience swallowing dysfunction (dysphagia) acutely [1]. Fortunately, the majority of them will recover swallowing

A. Vose $\cdot$ J. Nonnenmacher $\cdot$ M. L. Singer .

M. González-Fernández

Department of Physical Medicine and Rehabilitation, Johns

Hopkins University School of Medicine, Baltimore, USA

M. González-Fernández ( $₫)$

Physical Medicine and Rehabilitation, Johns Hopkins University School of Medicine, 600 North Wolfe St. Phipps 186, Baltimore, MD 21287, USA

e-mail: mgonzal5@jhmi.edu function within 7 days [2]. Approximately $11-13 \%$ will continue to have dysphagia at 6 months [3]. This represents 80,000 of the 665,000 new stroke survivors each year in the US [4]. Dysphagia is not only a risk factor for malnutrition, dehydration, and pneumonia after stroke, but also has a profound impact on stroke survivors discharge location; $60 \%$ of non-dysphagic patients are discharged home after a stroke versus only $21 \%$ of patients with dysphagia [5].

Early treatment of dysphagia aims to reduce secondary complications such as dehydration, malnutrition, and pneumonia and allow for spontaneous recovery of swallowing function. For those with dysphagia persisting beyond the acute phase, it is crucial to continue treatment that, in addition to reducing secondary complications, targets the physiologic deficits caused by the stroke with the goal of improving swallowing function or compensating for lost function.

\section{Dysphagia Diagnosis}

Stroke patients should be screened for dysphagia followed by formal evaluation for those failing screening evaluation. Controversy exists as to the best method to screen or assess dysphagia after a stroke. Multiple screening protocols have been proposed (See Ref. [6 ${ }^{\bullet}$ ] for a summary). Formal evaluation primarily relies on bedside evaluations performed by speech language pathologists but may also include instrumental assessment using videofluoroscopy (VFSS) or videoendoscopy (FEES). The presence of dysphonia, dysarthria, abnormal gag reflex, abnormal voluntary cough, voice change with swallowing, and cough with swallowing has been described as suggestive of increased aspiration risk [7, 8]. The challenge in screening or 
assessing swallowing dysfunction after stroke is that a large proportion of stroke patients with dysphagia will aspirate silently, i.e., will not demonstrate signs of airway invasion during feeding [9]. Thus, some experts in this area suggest that instrumental assessment is necessary to detect silent aspiration. Another goal of instrumental assessment is to identify the physiologic impairments resulting is swallowing dysfunction to allow for targeted interventions.

\section{Stroke Location and Physiologic Deficits}

Normal control of the swallow involves multiple areas of the brain: brain stem, thalamus, basal ganglia, limbic system, cerebellum, and motor and sensory cortices among others $[10,11]$. If any of these areas are damaged by stroke, serious complications, including dysphagia, can occur. A report by Daniels et al. suggests that lesions disrupting cortical-subcortical connectivity are more likely to increase the risk of aspiration in stroke patients as compared to isolated cortical or subcortical lesions, and that intra-hemispheric locations appear to be more critical than hemisphere or lesion size in predicting dysphagia severity and risk of aspiration [10].

Timing of the swallowing phases, swallowing initiation, and airway protection is regulated by sensory input to the swallowing central pattern generator (CPG) in the brain stem [12-14]. Brainstem strokes, especially lateral medullary strokes, often result in severe, global dysphagia which results in aspiration $[13,15]$. Damage to this area can result in weakness or paralysis of the ipsilateral pharynx, larynx, and soft palate which negatively impacts timing and coordination of the pharyngeal swallow and upper esophageal sphincter (UES) control [13, 15]. Lateral medullary strokes may also cause ataxia and reduced temperature sensation [16].

Dysphagia related to dysfunction of supratentorial structures is the most common type seen in neurological disease. In stroke, the size of the unaffected swallowing cortical area predicts dysphagia symptoms [17]. The cerebral cortex is involved in the regulation and execution of the motor response and of sensorimotor control that may result in complex deficits of movement in the absence of weakness [18]. Motor regulation, execution, and sensorimotor control have also been associated with the primary motor, motor supplementary, and primary somatosensory cortices [19-21]. Disruptions of cortical motor input result in impairment of the voluntary initiation of deglutition [22, 23]. The internal capsule is important for the relay of information from the brain stem to the cortex. Strokes in the area of the internal capsule may result in acute disconnection between the cortical swallowing centers and the CPG [13, 21, 24].
Traditional views suggest that lesions must occur in the brainstem or bilaterally in the cerebral cortex to produce dysphagia; however, ongoing research has focused on the laterality of stroke lesions as well as differences in anterior and posterior locations. Daniels concluded that lesions associated with dysphagia were more likely to be anterior to the central sulcus and cortical rather than purely subcortical in location [18]. Research by Hamdy et al. suggests the possibility of unilateral hemispheric dominance which varies between individuals [14].

Multiple studies have described longer duration of pharyngeal transit and increased pharyngeal retention in patients with right hemisphere damage resulting in higher risk of laryngeal penetration and aspiration when compared to patients with damage in the left hemisphere $[10,25]$. Patients with right hemisphere dysfunction may have a greater need for non-oral nutrition due to significant pharyngeal dysmotility with all consistencies [10]. Hamdy et al. stated that the pharyngeal phase of swallowing is the most important clinical determinant of aspiration in stroke populations [17]. Studies by Robbins and Levine [26] and Steinhagen et al. suggest that left hemispheric damaged patients show oral motility dysfunction with reduced coordination of lingual musculature [27]. This results in poor bolus organization, delayed oral transit, and lateral sulci retention of the bolus [16].

Several studies have identified the insular cortex as one of the most common sites of involvement when dysphagia occurs as a result of stroke. It has been suggested that it may be responsible for the organization of complex behaviors related to the face and mouth [27, 28]. Insular infarction can result in prolonged dysphagia and cause sympathetic hyperactivity $[27,29,30]$. However, focal lesions in this region are uncommon because of the area's vascular supply [10]. While other cortical areas have been implicated in swallowing, including the anterior cingulate, orbitofrontal cortex, and temporopolar cortex, additional research is needed to determine the functional impact of lesions in these areas on swallowing [21, 31, 32].

\section{Traditional Dysphagia Therapy}

The selection of any strategy or treatment option for patients with dysphagia should be based on the clinician's experience, the patient's desires and the best available evidence from published literature. After critical review of the current literature supporting therapeutics used to treat swallowing disorders, the most current and best available evidence for each technique and exercise is summarized in the section below. Therapeutic techniques were divided into those used as compensatory strategies, exercises, and those used as both compensatory strategies and/or exercises. Decisions about 
therapeutic techniques implemented should be based on identification of impaired physiology seen during instrumental evaluation (i.e., VFSS and/or FEES).

Compensatory swallowing strategies are often used by clinicians where the goal is not to change swallowing physiology but instead the goal is to prevent symptoms of dysphagia in order to maintain safety and ensure adequate nutrition and hydration. These techniques are by definition compensatory and do not result in long-term physiologic changes. These include volume and texture modifications as well as strategies such as chin tuck, head tilt, head turn, and chin tuck and head turn.

Swallowing exercises are often used to treat dysphagia with the goal of altering swallowing physiology and promoting long-term changes. Exercises are expected to impact swallowing mechanics and impact bolus flow. Some maneuvers may serve as a compensatory strategy and also function as rehabilitative exercises such as the effortful swallow, supraglottic swallow, super-supraglottic swallow, and the Mendelsohn maneuver. Other exercises may be used which are not compensatory and are meant to solely improve swallowing physiology such as the Shaker exercise and Masako (tongue hold) maneuver.

A combination of compensatory strategies and rehabilitative strategies may be implemented to both manage dysphagia symptoms and improve swallowing physiology, depending on the physiologic impairment identified during a FEES and/or VFSS. For patients with dysphagia secondary to stroke, regardless of stroke etiology, the goal is to identify and treat the physiologic impairments. By relating treatment to physiology, the goal is to improve outcomes and alleviate dysphagia symptoms. Described below are treatments used to manage dysphagia, including their purpose, instructions and impact on swallowing physiology. Table 1 summarizes traditional treatment techniques and the physiologic impairments they target. The effectiveness of each of these interventions vary, thus implementation during VFSS or FEES will allow clinicians to determine which interventions will be safe and effective in minimizing risks, optimizing nutrition and hydration, and treating the underlying physiologic deficits.

\section{Compensatory Techniques}

\section{Chin Tuck (Head Flexion)}

The chin tuck (head flexion) has been a technique used for patients who have decreased airway protection associated with delayed swallow initiation and/or reduced tongue base retraction. Patients are instructed to "bring their chin to their chest" and maintain this posture throughout the duration of the swallow [33]. Physiologic changes of the chin tuck observed in fluoroscopy as compared to normal swallows with head in neutral position include expansion of vallecular recesses, approximation of tongue base toward pharyngeal wall, narrowing entrance to the laryngeal vestibule, reduction in distance between hyoid and larynx, and increased duration of swallowing apnea during the swallow [33, 34].

\section{Head Rotation (Head Turn)}

Head rotation is a compensatory strategy used for patients with unilateral pharyngeal and/or laryngeal weakness as well as reduced UES opening. Patients may be instructed to "turn your head to the side as if you are looking over your shoulder." Head rotation toward the side of impairment effectively redirects the bolus to the side of the pharynx opposite the rotation (the stronger side) [35]. Head rotation is also a beneficial technique which drops UES pressure on the side opposite to the head turn thus allowing for increased extension and duration of UES opening. For patients with reduced laryngeal closure, head rotation narrows the laryngeal entrance and increases vocal fold closure by applying extrinsic pressure which may be beneficial for patients with reduced laryngeal adduction in the case of unilateral vocal fold impairment [35-37].

\section{Head Tilt}

The head tilt is used for patients with unilateral oral weakness. Patients are instructed to "tilt your head like you're trying to touch your ear to your shoulder." This technique is beneficial as it directs the bolus to the stronger side of the oral cavity [38].

\section{Bolus Viscosity, Texture, and Volume Modifications}

Increasing the volume and/or viscosity for liquids is another technique used to reduce dysphagia symptoms for some patients. Thickening liquids may be used for patients who have poor oral control of thin liquids and/or demonstrate reduced airway protection. Physiologically, studies have shown that with increasing bolus viscosity there is an increase in lingual-palatal contact pressure, pharyngeal pressure and UES relaxation, and slowing of bolus transit [39-42]. Alternatively, increasing bolus volume increases bolus transit time as exemplified by sustained laryngeal elevation and hyoid excursion. Additionally, during larger volume swallows there is increased laryngeal closure duration, increased duration of UES opening as well as decreased duration of tongue base contact to posterior pharyngeal wall [43-45]. In addition to altering liquid viscosity, some patients may benefit from texture-modified foods. Aside from the social and personal reasons patients may alter their food texture; patients may benefit from 


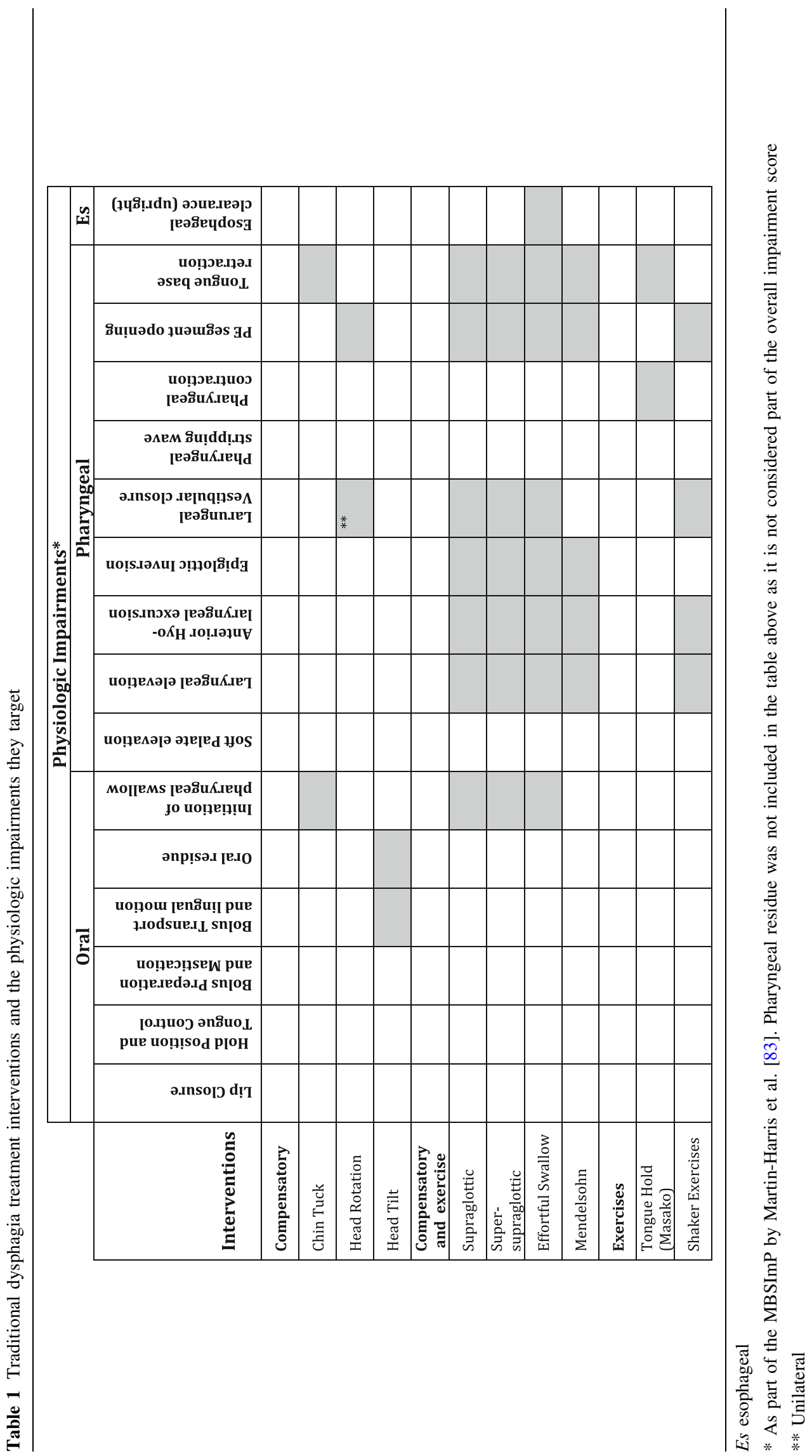


texture alterations in the setting of poor dentition, reduced lingual function, and/or increased aspiration risk.

\section{Compensatory and Exercise}

\section{Supraglottic Swallow}

The supraglottic swallow is used for patients who demonstrate reduced airway protection during the swallow. Instructions provided are to: "First, inhale deep, then hold your breath, continue to hold your breath and swallow, immediately after you swallow (before you inhale), cough then immediately swallow again" [46]. The physiologic benefits of this strategy include increased airway closure by increasing arytenoid approximation and true vocal fold closure as well is increasing UES opening during the swallow. Additionally, the airway is protected earlier in the swallow and hyolaryngeal excursion is prolonged which may be beneficial for patients with delayed swallow initiation [46-49].

\section{Super-Supraglottic Swallow}

Similar to the supraglottic swallow, the super-supraglottic swallow is also used for patients with reduced airway closure; however, the difference with the super-supraglottic is patients are instructed to implement an effortful breath hold, "take a breath and hold it tightly while bearing down; continue to hold your breath and bear down as you swallow; immediately after your swallow (before you inhale) cough then immediately swallow hard again (before you inhale)" [46]. With the supraglottic swallow, the breath hold requires no increased effort or bearing down. Physiologically with this technique, the patient has earlier tongue base movement, higher hyoid position at swallow onset, increased hyoid movement as well as longer bolus transit time, tongue base and pharyngeal wall contact, and airway closure [46, 48, 49]. Both the supraglottic swallow and the supra-supraglottic swallow maneuvers may result in Valsalva. A study by Chaudhuri et al. demonstrated that using these maneuvers in stroke patients resulted in arrhythmias that occurred within a treatment session, subsided within minutes of session end, and that did not occur during other activities [50]. Thus, clinicians should be mindful of using these maneuvers in stroke patients especially in those with coexisting heart disease.

\section{Effortful Swallow}

The effortful swallow is used for patients who present with clinically significant residue in the valleculae and/or pyriform sinuses as well as for patients who may have decreased airway closure. Instructions are to "squeeze your throat muscles as hard as you can while swallowing" [51]. Physiologically, the effortful swallow increases hyolaryngeal excursion, duration of hyoid elevation and UES opening, laryngeal closure, lingual pressures, peristaltic amplitudes in the distal esophagus, and pressure and duration of tongue base retraction [39, 45, 47, 52-54].

\section{Mendelsohn Maneuver}

The Mendelsohn maneuver is a technique used for patients with decreased hyolaryngeal excursion and/or decreased duration of UES opening. Prior to instructions, it is suggested that patients should first feel laryngeal elevation by palpation of thyroid cartilage during swallows. Instructions are then given to: "Swallow and when you feel your thyroid cartilage elevate hold it there for several seconds before finishing the swallow" [51]. This technique increases extent and duration of hyolaryngeal excursion, UES opening, pharyngeal peak contractions, bolus transit time and duration, and pressure of tongue base contact [ 45 , $51,55-57]$.

\section{Exercises}

\section{Tongue Hold}

The tongue hold is used for reduced tongue base, and pharyngeal wall contact. Instructions are provided to, "hold the anterior tongue (slightly posterior to the tongue tip) between the teeth while swallowing" [58]. Physiologically, the exercise increases anterior bulging of the posterior pharyngeal wall [58-60].

\section{Shaker Exercise}

The Shaker Exercise is used for patients who have decreased UES opening and weakness of the suprahyoid muscles. Instructions are to: "lie in the supine position; complete 3 head lifts sustained for $1 \mathrm{~min}$ each; 1 min rest period between each head lift; then complete 30 consecutive head lifts holding for $2 \mathrm{~s}$ each" [61]. The suggested frequency is three times each day for 6 consecutive weeks. Physiologically, the exercise increases anterior hyolaryngeal excursion, UES opening, strengthens suprahyoid muscles, and enhances thyrohyoid shortening [61-65].

\section{Other Interventions}

In addition to traditional dysphagia management as discussed above, there are alternate treatment modalities for individuals suffering from dysphagia following a stroke. Some of the most salient ones are reviewed below. 
Neuromuscular Electrical Stimulation (NMES)

Neuromuscular electrical stimulation (NMES) is a treatment where electrodes are placed on the anterior neck and an electrical current evokes a muscle contraction. NMES treatment is typically used as an adjunct modality concurrently while the patient swallows and/or performs a traditional exercise. There are currently two commercially available NMES devices, VitalStim, which was approved by the US Food and Drug Administration in 2002, and eSwallow, which was introduced and approved in 2011.

There are mixed views and research findings on incorporating NMES into dysphagia management practices. In a study by Xia et al. [66], 120 post-stroke patients who exhibited dysphagia were randomly assigned to receive either traditional swallowing treatment, solely NMES, or NMES in conjunction with traditional swallowing management [66]. The researchers found that the individuals who received NMES along with traditional treatment made significantly greater improvements in all four-outcome measures as opposed to the group who received traditional treatment alone or the group who received NMES alone. Another study by Kushner et al. examined the effect of NMES use with feeding tube dependent patients following an acute stroke, during inpatient rehabilitation [67]. The study compared the efficacy of NMES in addition to traditional dysphagia therapy (including progressive resistance training) with that of traditional dysphagia therapy and progressive resistance training alone. The researchers concluded that the addition of NMES was significantly more effective than traditional dysphagia management alone in reducing feeding tube dependent dysphagia in patients following an acute stroke. Other research confirms that use of thermal-tactile stimulation in conjunction with NMES is better for patients with dysphagia following a stroke as compared to thermal-tactile stimulation alone [68].

Conversely, other researchers have questioned the use of NMES for the treatment of dysphagia. Some researchers conclude NMES can actually decrease swallow function. In a study of swallowing physiology by Ludlow et al. hyoid movement and use of NMES was measured in patients with severe and chronic dysphagia [69]. When NMES was applied at the maximum level tolerated, hyoid depression occurred at rest in some of the patients in the study. Due to hyolaryngeal depression during NMES, it may be beneficial if a patient can overcome the resistance. However, the inability to swallow against the resistive lowering of the hyolaryngeal mechanism places severely dysphagic patients at increased risk for penetration and/or aspiration [70]. Surface NMES activates all tissues that can be stimulated by its electrical current and thus lacks specificity. Ultimately NMES related to dysphagia management must not be viewed as "one size fits all." Clinicians must therefore continue to follow the research to determine the most appropriate and effective use of this treatment modality for patients with dysphagia based on the individual's unique variables [71•]. Additional research is currently being conducted to advance NMES for improved dysphagia treatment opportunities.

\section{Oral Stimulation and Other Interventions}

In addition to NMES, many clinicians use oral exercises (to include tactile-thermal stimulation, lingual, and labial strengthening) as a treatment modality for stroke patients with dysphagia. The application of oral sensory stimulation for treatment of dysphagia is somewhat controversial; however, there are data that suggest it may increase corticobulbar excitability, which has been associated with swallowing recovery after a stroke [72]. One study assessed the effects of stimulation to the faucial pillars in 16 hemispheric stroke patients with a diagnosis of dysphagia. The patients' swallowing was assessed before, and $60 \mathrm{~min}$ after $0.2 \mathrm{~Hz}$ electrical or sham stimulation. Various swallowing measures were assessed by videofluoroscopy to include laryngeal closure and pharyngeal transit time. Initiation of laryngeal closure was delayed in both groups prior to intervention. However, the researchers found that compared to baseline, there was no functional change in swallowing physiology among the two subject groups.

Ice massage is also discussed and compared to tactilethermal stimulation in the literature as a prefeeding technique to facilitate dry swallows as well as for initiating the pharyngeal swallow trigger [73]. With this technique, massage with an ice stick is applied to the throat, base of the anterior faucial arches, base of tongue, and the posterior pharyngeal wall for $10 \mathrm{~s}$ with rubbing and light compression. Ice massage shortened the latency for triggering the swallow after the command and the massage in itself had an immediate effect on triggering a swallow response even in patients who could not swallow voluntarily. The authors of this research propose that this technique is more advantageous than tactile-thermal stimulation as it can be conducted simply and inexpensively with a frozen water impregnated cotton tipped stick and it can be conducted without the need for a voluntary swallow following stimulation. The effectiveness of ice massage was found to be more significant in subjects with supranuclear lesions than in those with nuclear lesions. Ice massage could activate a damaged supranuclear tract of swallowing and/or a normal nuclear and subnuclear tract.

Additionally, lip muscle training has been studied in stroke patients with dysphagia. Hagg and Anniko conducted a retrospective study of 30 stroke patients. The researchers used an oral screen, which was found to improve lip force as well as swallowing capacity in patients 
who exhibited oropharyngeal dysphagia [74]. These results were without reference to duration of prior treatment of dysphagia and/or the presence or absence of central facial paresis. Treatment results were stated to be attributable to sensory motor stimulation and the plasticity of the central nervous system as opposed to isolated training of the lip muscles.

Lingual exercises are another modality discussed in the literature. Robbins et al. initially examined the effects of lingual exercise on swallow recovery following stroke using the Iowa Oral Performance Instrument (IOPI) [75]. Ten stroke patients participated in an 8-week isometric lingual exercise program using the IOPI, by which an air filled bulb is compressed between the tongue and hard palate. Results revealed all ten individuals improved isometric and swallowing pressures as well as a reduction in airway invasion of thin liquids as measured via videofluoroscopic swallow study.

In addition to the IOPI, there is another product available that similarly addresses isometric lingual strength, referred to commercially as SwallowStrong (formerly MOST). As part of I-PRO (isometric progressive resistance oropharyngeal) therapy, this device uses a touch screen tablet that connects to a custom mouthpiece. The interface uses real time monitoring to assess lingual strength and create an individualized program for the patient.

There is strong evidence toward the effectiveness of tongue strengthening devices for the measurement of tongue strength and endurance. These devices may be beneficial as an evaluation tool and training device for speech pathologists that evaluate and treat patients with diminished lingual strength and endurance that contributes to dysphagia symptoms [76•]. Surface electromyography (sEMG) biofeedback is another treatment modality for dysphagia. Crary et al. discussed a retrospective analysis of 25 patients status post stroke who received a systematic program which was supplemented with sEMG. Ninety-two percent of the subjects experienced an increase in functional oral intake [77]. The use of sEMG appears to be a viable adjunct to traditional therapy measures for patients following stroke as patients who experience physiologic based dysphagia are more apt to benefit from a treatment modality that facilitates motor learning of swallowing movements.

Transcranial magnetic stimulation (TMS) as well as transcranial direct current [78•] stimulation (tDCS) are also discussed in the literature, and are used with increasing frequency in patients with post-stroke dysphagia. TMS is a method which has been used to explore the corticomotor physiology of many motor tasks, to include swallowing. TMS-induced motor evoked potentials have been documented to change the excitability of cortical projections to swallowing muscles. The link between swallowing physiology and cortical excitability remains unclear [78•].
Yang et al. studied sixteen post-stroke dysphagic patients as measured via videofluoroscopic swallowing studies (VFSS) [79]. The patients were assigned randomly to a sham group or to receive tDCS to the pharyngeal motor cortex of the affected hemisphere during the course of 30 min of traditional swallowing therapy for a 10-day time frame. The effect of tDCS on dysphagia was measured with the functional dysphagia scale (FDS) based on results of VFSS at baseline, immediately following treatment, and 3 months following intervention. Three months following intervention, the group who received tDCS experienced greater improvement on the FDS, opposed to the sham group. Comparisons were made after controlling for factors such as age, National Institutes of Health Stroke Scale Score (NIHSS), lesion size, baseline FDS score, and stroke onset time. A systematic review by Adeyemo et al. studied pre- and post-motor outcomes of individuals who have had dysphagia following a stroke and received repetitive TMS and/or tDCS treatments [80॰]. Although most of the reviewed studies did not provide outcomes for long-term results, post treatment data revealed consistent functional improvements in the stroke population. Humbert et al. studied the pathophysiology of delayed swallow initiation in individuals with neurogenic dysphagia, and its relationship with of the primary sensory motor region of cortex [81]. Preliminary findings revealed that transient disruption of the primary sensory-motor cortex can delay swallowing onset. These findings are significant as they provide insight regarding cortical control and involvement with swallowing initiation.

Other factors identified in the literature associated with progression of dysphagia management are oral hygiene, nutrition, and the patients' awareness of their swallowing disability [73].

\section{Oral Hygiene}

A stringent oral hygiene program is essential for patients at risk of aspiration given that the mouth is the most common location of bacteria. In addition to traditional interventions for dysphagia, all patients should include a comprehensive oral hygiene program to their therapy routine. Multiple studies have demonstrated a preventive effect of oral hygiene on pneumonia and other respiratory tract infections [82•].

\section{Conclusion}

Treatment of dysphagia after stroke should focus on the underlying physiologic deficits identified during formal swallowing evaluation. Traditional therapies focus on prevention of secondary complications early after stroke 
and continue into the sub-acute stage to improve affected swallowing control and mechanics. Novel therapies are promising, but research needs to continue with the goal to determine the best candidates, optimal dose, and frequency of such treatments.

\section{Compliance with Ethics Guidelines}

Conflict of Interest A Vose, J Nonnenmacher, ML Singer, and M González-Fernández all declare no conflicts of interest.

Human and Animal Rights and Informed Consent This article does not contain any studies with human or animal subjects performed by any of the authors.

\section{References}

Papers of particular interest, published recently, have been highlighted as:

- Of importance

1. Martino R, Foley N, Bhogal S, Diamant N, Speechley M, Teasell R. Dysphagia after stroke: incidence, diagnosis, and pulmonary complications. Stroke. 2005;36(12):2756-63.

2. Smithard DG, O'Neill PA, England RE, Park CL, Wyatt R, Martin DF, et al. The natural history of dysphagia following a stroke. Dysphagia. 1997;12(4):188-93.

3. Mann G, Hankey GJ, Cameron D. Swallowing function after stroke: prognosis and prognostic factors at 6 months. Stroke. 1999;30(4):744-8.

4. Stroke facts [Internet]. Available from: http://www.cdc.gov/ stroke/facts.htm.

5. Altman KW, Schaefer SD, Yu GP, Hertegard S, Lundy DS, Blumin $\mathrm{JH}$, et al. The voice and laryngeal dysfunction in stroke: a report from the neurolaryngology subcommittee of the american academy of otolaryngology-head and neck surgery. Otolaryngol Head Neck Surg. 2007;136(6):873-81.

6. - Gonzalez-Fernandez M, Ottenstein L, Atanelov L, Christian AB. Dysphagia after stroke: an overview. Curr Phys Med Rehabil Rep. 2013;1(3):187-96. This is a summary paper on dysphagia and provides details on evaluation beyond the scope of this article.

7. Ramsey DJ, Smithard DG, Kalra L. Early assessments of dysphagia and aspiration risk in acute stroke patients. Stroke. 2003;34(5):1252-7.

8. Daniels SK, Brailey K, Priestly DH, Herrington LR, Weisberg LA, Foundas AL. Aspiration in patients with acute stroke. Arch Phys Med Rehabil. 1998;79(1):14-9.

9. Splaingard ML, Hutchins B, Sulton LD, Chaudhuri G. Aspiration in rehabilitation patients: videofluoroscopy vs bedside clinical assessment. Arch Phys Med Rehabil. 1988;69(8):637-40.

10. Daniels SK, Foundas A, Iglesia G, Sullivan M. Lesion site in unilateral stroke patients with dysphagia. J Stroke Cerebrovasc Dis. 1996;6(1):30-4.

11. Zald H, Pardo J. The functional neuroanatomy of voluntary swallowing. Ann Neurol. 1999;46(3):281-6.

12. Jean A. Brain stem control of swallowing: neuronal network and cellular mechanisms. Physiol Rev. 2001;81(2):929-69.

13. Aydogdu I, Ertekin C, Tarlaci S, Turman B, Kiylioglu N, Secil Y. Dysphagia in lateral medullary infarction (wallenberg's syndrome): an acute disconnection syndrome in premotor neurons related to swallowing activity. Stroke. 2001;32(9):2081-7.
14. Hamdy S, Aziz Q, Rothwell JC, Hobson A, Barlow J, Thompson DG. Cranial nerve modulation of human cortical swallowing motor pathways. Am J Physiol. 1997;272(4 Pt 1):G802-8.

15. Kim H, Chung C, Lee K, Robbins J. Aspiration subsequent to a pure medullary infarction: lesion sites, clinical variables, and outcome. Arch Neurol. 2000;57(4):478-83.

16. Daniels SK, Schroeder MF, McClain M, Corey DM, Rosenbek JC, Foundas AL. Dysphagia in stroke: development of a standard method to examine swallowing recovery. J Rehabil Res Dev. 2006;43(3):347-56.

17. Hamdy S, Aziz Q, Rothwell JC, Crone R, Hughes D, Tallis RC, et al. Explaining oropharyngeal dysphagia after unilateral hemispheric stroke. Lancet. 1997;350(9079):686-92.

18. Daniels SK, Foundas AL. Lesion localization in acute stroke patients with risk of aspiration. J Neuroimaging. 1999;9(2):91-8.

19. Hamdy S, Rothwell JC, Brooks DJ, Bailey D, Aziz Q, Thompson DG. Identification of the cerebral loci processing human swallowing with H2(15)O PET activation. J Neurophysiol. 1999;81(4):1917-26.

20. Mosier K, Bereznaya I. Parallel cortical networks for volitional control of swallowing in humans. Exp Brain Res. 2001;140(3): $280-9$.

21. Gonzalez-Fernandez M, Kleinman J, Ky P, Palmer J, Hillis A. Supratentorial regions of acute ischemia associated with clinically important swallowing disorders: a pilot study. Stroke. 2008;39(11):3022-8.

22. Michou E, Hamdy S. Cortical input in control of swallowing. Curr Opin Otolaryngol Head Neck Surg. 2009;17(3):166-71.

23. Leopold NA, Daniels SK. Supranuclear control of swallowing. Dysphagia. 2010;25(3):250-7.

24. Mosier K, Patel R, Liu WC, Kalnin A, Maldjian J, Baredes S. Cortical representation of swallowing in normal adults: functional implications. Laryngoscope. 1999;109(9):1417-23.

25. Robbins J, Levine R, Maser A, Rosenbeck J, Kempster G. Swallowing after unilateral stroke of the cerebral hemisphere. Arch Phys Med Rehabil. 1993;74:1295-300.

26. Robbins J, Levine R. Swallowing after unilateral stroke of the cerebral cortex: preliminary experience. Dysphagia. 1988;3:11-7.

27. Steinhagen V, Grossman A, Benecke R, Walter U. Swallowing disturbance pattern relates to brain lesion location in acute stroke. Stroke. 2009;40:1903-6.

28. Mesulam M, Mufson E, editors. Cerbral cortex. 4th ed. New York: Plenum; 1985.

29. Broadley S, Croser D, Cottrell J, Creevy M, Teo E, Yiu D, et al. Predictors of prolonged dysphagia following acute stroke. J Clin Neurosci. 2003;10(3):300-5.

30. Tokgozoglu S, Batur M, Topcuoglu M, Saribas O, Kes S, Oto A. Effects of stroke localization on cardiac autonic balance and sudden death. Stroke. 1999;30:1307-11.

31. Hamdy S, Mikulis DJ, Crawley A, Xue S, Lau H, Henry S, et al. Cortical activation during human volitional swallowing: an eventrelated fMRI study. Am J Physiol. 1999;277(1 Pt 1):G219-25.

32. Martin RE, Goodyear BG, Gati JS, Menon RS. Cerebral cortical representation of automatic and volitional swallowing in humans. J Neurophysiol. 2001;85(2):938-50.

33. Welch MV, Logemann JA, Rademaker AW, Kahrilas PJ. Changes in pharyngeal dimensions effected by chin tuck. Arch Phys Med Rehabil. 1993;74(2):178-81.

34. Castell JA, Castell DO, Schultz AR, Georgeson S. Effect of head position on the dynamics of the upper esophageal sphincter and pharynx. Dysphagia. 1993;8(1):1-6.

35. Logemann JA, Kahrilas PJ, Kobara M, Vakil NB. The benefit of head rotation on pharyngoesophageal dysphagia. Arch Phys Med Rehabil. 1989;70(10):767-71.

36. Tsukamoto Y. CT study of closure of the hemipharynx with head rotation in a case of lateral medullary syndrome. Dysphagia. 2000;15(1):17-8. 
37. Ohmae Y, Ogura M, Karaho T, Kitahara S, Inouye T. Effects of head rotation on pharyngeal function during normal swallow. Ann Otol Rhinol Laryngol. 1998;107(4):344-8.

38. Logemann JA. Evaluation and treatment of swallowing disorders. 2nd ed. Austin TX: Pro-Ed; 1998.

39. Pouderoux P, Kahrilas PJ. Deglutitive tongue force modulation by volition, volume, and viscosity in humans. Gastroenterology. 1995;108(5):1418-26.

40. Chi-Fishman G, Sonies BC. Effects of systematic bolus viscosity and volume changes on hyoid movement kinematics. Dysphagia. 2002;17(4):278-87.

41. Dantas RO, Dodds WJ, Massey BT, Kern MK. The effect of highvs low-density barium preparations on the quantitative features of swallowing. AJR Am J Roentgenol. 1989;153(6):1191-5.

42. Butler SG, Stuart A, Castell D, Russell GB, Koch K, Kemp S. Effects of age, gender, bolus condition, viscosity, and volume on pharyngeal and upper esophageal sphincter pressure and temporal measurements during swallowing. J Speech Lang Hear Res. 2009;52(1):240-53.

43. Lazarus CL, Logemann JA, Rademaker AW, Kahrilas PJ, Pajak $\mathrm{T}$, Lazar R, et al. Effects of bolus volume, viscosity, and repeated swallows in nonstroke subjects and stroke patients. Arch Phys Med Rehabil. 1993;74(10):1066-70.

44. Bisch EM, Logemann JA, Rademaker AW, Kahrilas PJ, Lazarus CL. Pharyngeal effects of bolus volume, viscosity, and temperature in patients with dysphagia resulting from neurologic impairment and in normal subjects. J Speech Hear Res. 1994;37(5):1041-59.

45. Kahrilas PJ, Lin S, Logemann JA, Ergun GA, Facchini F. Deglutitive tongue action: volume accommodation and bolus propulsion. Gastroenterology. 1993;104(1):152-62.

46. Martin BJ, Logemann JA, Shaker R, Dodds WJ. Normal laryngeal valving patterns during three breath-hold maneuvers: a pilot investigation. Dysphagia. 1993;8(1):11-20.

47. Bülow M, Olsson R, Ekberg O. Videomanometric analysis of supraglottic swallow, effortful swallow, and chin tuck in patients with pharyngeal dysfunction. Dysphagia. 2001;16(3):190-5.

48. Donzelli J, Brady S. The effects of breath-holding on vocal fold adduction: implications for safe swallowing. Arch Otolaryngol Head Neck Surg. 2004;130(2):208-10.

49. Ohmae Y, Logemann JA, Kaiser P, Hanson DG, Kahrilas PJ. Effects of two breath-holding maneuvers on oropharyngeal swallow. Ann Otol Rhinol Laryngol. 1996;105(2):123-31.

50. Chaudhuri G, Hildner CD, Brady S, Hutchins B, Aliga N, Abadilla E. Cardiovascular effects of the supraglottic and supersupraglottic swallowing maneuvers in stroke patients with dysphagia. Dysphagia. 2002;17(1):19-23.

51. Kahrilas PJ, Logemann JA, Krugler C, Flanagan E. Volitional augmentation of upper esophageal sphincter opening during swallowing. Am J Physiol. 1991;260(3 Pt 1):G450-6.

52. Hind JA, Nicosia MA, Roecker EB, Carnes ML, Robbins J. Comparison of effortful and noneffortful swallows in healthy middle-aged and older adults. Arch Phys Med Rehabil. 2001;82(12):1661-5.

53. Huckabee M, Butler SG, Barclay M, Jit S. Submental surface electromyographic measurement and pharyngeal pressures during normal and effortful swallowing. Arch Phys Med Rehabil. 2005;86(11):2144-9.

54. Hiss SG, Huckabee ML. Timing of pharyngeal and upper esophageal sphincter pressures as a function of normal and effortful swallowing in young healthy adults. Dysphagia. 2005;20(2):149-56.

55. Jacob P, Kahrilas PJ, Logemann JA, Shah V, Ha T. Upper esophageal sphincter opening and modulation during swallowing. Dysphagia. 1989;97(6):1469-78.
56. Cook IJ, Dodds WJ, Dantas RO, Massey B, Kern MK, Lang IM, et al. Opening mechanisms of the human upper esophageal sphincter. Gastroenterology. 1989;257(5 Pt 1):G748-59.

57. Kahrilas PJ, Logemann JA, Lin S, Ergun GA. Pharyngeal clearance during swallowing: a combined manometric and videofluoroscopic study. Gastroenterology. 1992;103(1):128-36.

58. Fujiu M, Logemann JA, Pauloski BR. Increased postoperative posterior pharyngeal wall movement in patients with anterior oral cancer: Preliminary findings and possible implications for treatment. Am J Speech Lang Pathol. 1995;4(2):24-30.

59. Fujiu-Kurachi M, Fujiwara S, Tamine K, Kondo J, Minagi Y, Maeda Y, et al. Tongue pressure generation during tongue-hold swallows in young healthy adults measured with different tongue positions. Dysphagia. 2014;29(1):17-24.

60. Fujiu-Kurachi M. Developing the tongue holding maneuver. Dysphagia. 2002;11(1):9-11.

61. Shaker R, Kern M, Bardan E, Taylor A, Stewart ET, Hoffmann $\mathrm{RG}$, et al. Augmentation of deglutitive upper esophageal sphincter opening in the elderly by exercise. Am J Physiol. 1997;272(6 Pt 1):G1518-22.

62. Mepani R, Antonik S, Massey B, Kern M, Logemann J, Pauloski $\mathrm{B}$, et al. Augmentation of deglutitive thyrohyoid muscle shortening by the shaker exercise. Dysphagia. 2009;24(1):26-31.

63. Shaker R, Easterling C, Kern M, Nitschke T, Massey B, Daniels $\mathrm{S}$, et al. Rehabilitation of swallowing by exercise in tube-fed patients with pharyngeal dysphagia secondary to abnormal UES opening. Gastroenterology. 2002;122(5):1314-21.

64. Ferdjallah M, Wertsch JJ, Shaker R. Spectral analysis of surface electromyography (EMG) of upper esophageal sphincter-opening muscles during head lift exercise. J Rehabil Res Dev. 2000;37(3):335-40.

65. Easterling C, Grande B, Kern M, Sears K, Shaker R. Attaining and maintaining isometric and isokinetic goals of the shaker exercise. Dysphagia. 2005;20(2):133-8.

66. Xia W, Zheng C, Lei Q, Tang Z, Hua Q, Zhang Y, et al. Treatment of post-stroke dysphagia by vitalstim therapy coupled with conventional swallowing training. J Huazhong Univ Sci Technol Med Sci. 2011;31(1):73-6.

67. Kushner DS, Peters K, Eroglu ST, Perless-Carroll M, JohnsonGreene D. Neuromuscular electrical stimulation efficacy in acute stroke feeding tube-dependent dysphagia during inpatient rehabilitation. Am J Phys Med Rehabil. 2013;92(6):486-95.

68. Lim KB, Lee HJ, Lim SS, Choi YI. Neuromuscular electrical and thermal-tactile stimulation for dysphagia caused by stroke: A randomized controlled trial. J Rehabil Med. 2009;41(3):174-8.

69. Ludlow CL, Humbert I, Saxon K, Poletto C, Sonies B, Crujido L. Effects of surface electrical stimulation both at rest and during swallowing in chronic pharyngeal dysphagia. Dysphagia. 2007;22(1):1-10.

70. Ludlow CL. Electrical neuromuscular stimulation in dysphagia: current status. Curr Opin Otolaryngol Head Neck Surg. 2010;18(3):159-64.

71. - Humbert IA. Point counterpoint: Electrical stimulation for dysphagia. Perspectives on Swallowing and Swallowing Disorders. Dysphagia. 2011;20:102-108. This article provides information on the controversies surrounding the use of NMES for dysphagia rehabilitation.

72. Power ML, Fraser CH, Hobson A, Singh S, Tyrrell P, Nicholson DA, et al. Evaluating oral stimulation as a treatment for dysphagia after stroke. Dysphagia. 2006;21(1):49-55.

73. Parker C, Power M, Hamdy S, Bowen A, Tyrrell P, Thompson DG. Awareness of dysphagia by patients following stroke predicts swallowing performance. Dysphagia. 2004;19(1):28-35.

74. Hagg M, Anniko M. Lip muscle training in stroke patients with dysphagia. Acta Otolaryngol. 2008;128(9):1027-33. 
75. Robbins J, Kays SA, Gangnon RE, Hind JA, Hewitt AL, Gentry LR, et al. The effects of lingual exercise in stroke patients with dysphagia. Arch Phys Med Rehabil. 2007;88(2):150-8.

76. - Adams V, Mathisen B, Baines S, Lazarus C, Callister R. A systematic review and meta-analysis of measurements of tongue and hand strength and endurance using the iowa oral performance instrument (IOPI). Dysphagia. 2013;28(3):350-69. Provides a summary of tongue strength and endurance measures.

77. Crary MA, Carnaby Mann GD, Groher ME, Helseth E. Functional benefits of dysphagia therapy using adjunctive sEMG biofeedback. Dysphagia. 2004;19(3):160-4.

78. - Macrae PR, Jones RD, Huckabee ML. The effect of swallowing treatments on corticobulbar excitability: a review of transcranial magnetic stimulation induced motor evoked potentials. J Neurosci Methods. 2014;233C:89-98. A review on TMS in the context of dysphagia.

79. Yang EJ, Baek SR, Shin J, Lim JY, Jang HJ, Kim YK, et al. Effects of transcranial direct current stimulation (tDCS) on poststroke dysphagia. Restor Neurol Neurosci. 2012;30(4):303-11.
80. - Adeyemo BO, Simis M, Macea DD, Fregni F. Systematic review of parameters of stimulation, clinical trial design characteristics, and motor outcomes in non-invasive brain stimulation in stroke. Front Psychiatry. 2012;3:88. This article provides guidance as to the use of noninvasive brain stimulation after stroke.

81. Humbert IA, Poletto CJ, Saxon KG, Kearney PR, Crujido L, Wright-Harp W, et al. The effect of surface electrical stimulation on hyolaryngeal movement in normal individuals at rest and during swallowing. J Appl Physiol. 2006;101(6):1657-63.

82. - Sorensen RT, Rasmussen RS, Overgaard K, Lerche A, Johansen AM, Lindhardt T. Dysphagia screening and intensified oral hygiene reduce pneumonia after stroke. J Neurosci Nurs. 2013;45(3):139-46. Guidelines are provided for dysphagia screening and the use of an oral hygiene program to reduce morbidity after stroke.

83. Martin-Harris B, Brodsky MB, Michel Y, Castell DO, Schleicher M, Sandidge J, et al. MBS measurement tool for swallow impairment-MBSImp: establishing a standard. Dysphagia. 2008;23:392-405. 